\title{
A Remark on Arbitrage and Martingale Measure
}

\author{
By
}

\author{
Shigeo KusUOKA*
}

\section{$\S 0$. Introduction}

In the option pricing theory, as was pointed out in Harrison-Kreps [6], the most important fact is that the absence of arbitrage follows from the existence of equivalent martingale measure for the price process of securities. There are several attempts to show the converse statement that the absence of arbitrage implies the existence of an equivalent martingale measure. In the discrete time case, the proof for the most general case has been given by Dalang-Morton-Willinger [2] and Schachermayer [9]. On the continuous time case Stricker [11] gave beautiful results, and they were extended by Delbaen [3], Schachermayer [10] and Delbaen-Schachermayer [4], [5]. In particular, the Mackey topology is cleverly used in Delbaen [3], and his result is quite satisfactory in the case where price processes are path-wise continuous.

In this paper, we give some remarks on Orlicz spaces, Mackey topologies, and results by Ansel-Stricker [1] and Delbaen [3]. Then we give a certain necessary and sufficient conditions for the existence of an equivalent martingale measure.

\section{Acknowledgement}

This paper is quite different from the original version. The original results were fully refined and improved by Professors F. Delbaen, W. Schachermayer and $\mathrm{C}$. Stricker. The author thanks them for their useful comments. In particular, they pointed out that the original result was related to Orlicz spaces and Mackey topology. Theorem (3.2) is due to them. The author is very grateful to Prof. H. Föllmer for useful discussions. He also informed the author on Stricker's results and showed the original results to these three professors.

Received November 5, 1992.

1991 Mathematics Subject Classifications: 90A09, 60G44, 46E30.

* Research Institute for Mathematical Sciences, Kyoto University, 606-01 Kyoto, Japan. Current Address: Department of Mathematical Sciences, University of Tokyo, 7-3-1 Hongo, Tokyo, 113, Japan. 


\section{$\S 1$. Orlicz Spaces and Extension of Yan's Theorem}

(1.1) Definition. We say that $F:[0, \infty) \rightarrow[0, \infty)$ is a Young function, if (i) $F$ is continuously differentiable,

(ii) $F(0)=F^{\prime}(0)=0$,

and

(iii) $F^{\prime}$ is strictly increasing function and $\lim _{t \uparrow \infty} F^{\prime}(t)=\infty$.

Remark. In some books (e.g. [8], [13]), the more general definition are used as the definition of Young functions.

For any Young function $F:[0, \infty) \rightarrow[0, \infty)$, let $\Phi_{F}:[0, \infty) \rightarrow[0, \infty)$ be given by $\Phi_{F}(y)=\int_{0}^{y}\left(F^{\prime}\right)^{-1}(t) d t, \quad y \geqq 0$, where $\left(F^{\prime}\right)^{-1}$ is the inverse function of $F^{\prime}$. Then it is easy to see that $\Phi_{F}$ is also a Young function. We call $\Phi_{F}$ the complementary Young function of $F$.

The following is well-known (see [8] for example).

(1.2) Proposition. Let $F:[0, \infty) \rightarrow[0, \infty)$ be a Young function and $G:[0, \infty)$ $\rightarrow[0, \infty)$ be its complementary Young function of $F$. Then

$$
G(y)=\max \{x y-F(y) ; y \geqq 0\}, \quad x \geqq 0 .
$$

Let $(\Omega, \mathscr{B}, P)$ be a probability space. Let $\sim$ be the usual equivalence relations for random variables such that $X \sim Y$ if $X=Y P$-a.s. For each Young function $F$, we introduce

$$
L_{F}^{0}=\{\xi ; \xi \text { is a random variable, } E[F(a|\xi|)]<\infty \text { for any } a>0\} / \sim
$$

and

$$
L_{F}=\{\xi ; \xi \text { is a random variable, } E[F(a|\xi|)]<\infty \text { for some } a>0\} / \sim
$$

Clearly $L_{F}^{0} \subset L_{F}$. We define a norm \|\|$_{F}$ on $L_{F}$ by

$$
\|\xi\|_{F}=\inf \left\{a>0 ; E\left[F\left(a^{-1}|\xi|\right)\right] \leqq 1\right\} .
$$

The space $L_{F}$ is called the Orlicz space.

Then the following is known. (See Krasnosel'skii and Rutickii [8] and Zaanen [13] for example).

(1.3) Proposition. Let $F:[0, \infty) \rightarrow[0, \infty)$ be a Young function and $G:[0, \infty)$ $\rightarrow[0, \infty)$ be its complementary Young function. Then we have the following.

(1) $L_{F}$ is a Banach space with the norm $\|\cdot\|_{F}$.

(2) $L_{F}^{0}$ is the closure of $L^{\infty}(\Omega, \mathscr{B}, P)$ in $L_{F}$.

(3) $E\left[F\left(\|\xi\|_{F^{-1}} \xi\right)\right] \leqq 1$ for any $\xi \in L_{F} \backslash\{0\}$.

(4) $E\left[F\left(\|\xi\|_{F}{ }^{-1} \xi\right)\right]=1$ for any $\xi \in L_{F}^{0} \backslash\{0\}$. 
(5) $E[|\xi \eta|] \leqq 2\|\xi\|_{F}\|\eta\|_{G}, \xi \in L_{F}, \eta \in L_{G}$.

(6) If $\varphi: L_{F}^{n} \rightarrow \boldsymbol{R}$ is a continuous linear function, then there is a unique $\eta \in L_{G}$ such that

$$
\varphi(\xi)=E[\hat{\xi} \eta], \quad \xi \in L_{F}^{0} .
$$

(7) If $\eta \in L_{G}$, then $\varphi: L_{F}^{0} \rightarrow \boldsymbol{R}$ given by $\varphi(\xi)=E[\xi \eta]$, $\xi \in L_{F}^{n}$, is a continuous linear function, and

$$
\|\eta\|_{G} \leqq\|\varphi\|_{\left(L_{F}\right) *} \leqq 2\|\eta\|_{G} .
$$

Since $L_{F}$ is a Banach lattice and any positive operator in $L_{F}$ is a continuous linear operator (e.g. Theorem 83.12 in [13]). So we have the following from Proposition (1.3) (6).

(1.4) Corollary. Let $F:[0, \infty) \rightarrow[0, \infty)$ be a Young function and $G:[0, \infty) \rightarrow$ $[0, \infty)$ be its complementary Young function. If $\varphi: L_{F}^{\prime \prime} \rightarrow \boldsymbol{R}$ is a linear operator such that $\varphi(\xi) \geqq 0$ for any $\xi \in L_{F}^{0}$ with $\xi \geqq 0$ P-a.s., then there is an $\eta \in L_{G}$ such that $\eta \geqq 0$ P-a.s. and $\varphi(\xi)=E[\xi \eta], \xi \in L_{F}^{\prime \prime}$.

(1.5) Theorem. Let $K$ be a convex set in $L^{\infty}(\Omega, \mathscr{B}, P)$ with $0 \in K$. Let $F:[0, \infty)$ $\rightarrow[0, \infty)$ be a Young function and $G:[0, \infty) \rightarrow[0, \infty)$ be its complementary Young function. Then the following three conditions are equivalent.

(1) If $\xi \in L_{\perp}^{\infty}$ and $P(\xi>0)>0$, then $\{c \cdot \xi ; c>0\} \not \subset \overline{K-L_{\top}^{\infty} F^{\prime}}$. Here $L_{+}^{\infty}=$ $\left\{\eta \in L^{\infty}(\Omega, \mathscr{B}, P) ; \eta \geqq 0\right.$ P-a.s. $\}$ and $\overline{K-L_{-}^{\infty}} F$ is the closure of $K-L_{+}^{\infty}$ in $L_{F}^{0}$ with respect to \|\|$_{F-\text { topology. }}$

(2) If $A \subseteq \mathscr{B}$ and $P(A)>0$, then $\left\{c \cdot 1_{A} ; c>0\right\} \not \subset \overline{K-L_{\vdash}^{\infty} \text {. }}$.

(3) There is a $\rho \in L_{G}$ such that $\rho>0$ P-a.s., $E[\rho]=1$, and $\sup _{\xi \in K^{-}} E[\xi \rho]<\infty$.

Proof. The proof is exactly the same as the proof of Theorem 1 in AnselStricker [1], if we note Proposition (1.3) (6) and (7) (which imply the fact that $L_{G}$ is the dual space of $\left.L_{F}^{0}\right)$.

\section{§2. A Remark on Mackey Topology}

Now we introduce the following notions.

(2.1) Definition. (1) Let $\left\{\xi_{n}\right\}_{n=1}^{\infty}$ be a sequence in $L_{F}$ and $\xi \in L_{F}$. We say that $\xi_{n} \underset{w}{\stackrel{F}{\rightarrow}} \xi, n \rightarrow \infty$, if

(i) $\xi_{n}$ converges to $\xi$ in probability as $n \rightarrow \infty$,

and

(ii) $\sup _{n} E\left[F\left(a\left|\xi_{n}\right|\right)\right]<\infty$ for some $a>0$.

(2) Let $\left\{\xi_{n}\right\}_{n=1}^{\infty}$ is a sequence in $L_{F}^{0}$ and $\xi \in L_{F}^{0}$. We say that $\xi_{n} \stackrel{F}{\rightarrow} \xi, n \rightarrow \infty$, if 
(i) $\xi_{n}$ converges to $\xi$ in probability as $n \rightarrow \infty$, and

(ii) $\sup _{n} E\left[F\left(a\left|\xi_{n}\right|\right)\right]<\infty$ for all $a>0$.

(3) For any subset $A$ in $L_{F}^{0}, C l_{F}(A)=\left\{\xi \in L_{F}^{0}\right.$; there is a sequence $\left\{\xi_{n}\right\}_{n=1}^{\infty}$ $\subset A$ such that $\left.\xi_{n} \stackrel{F}{\rightarrow} \xi, n \rightarrow \infty\right\}$.

Then we have the following.

(2.2) Proposition. If $\xi, \xi_{1}, \xi_{2}, \cdots \in L_{F}$ and $\xi_{n} \rightarrow \xi$ in \|\|$_{F}$, then $\underset{\xi_{n}}{\stackrel{F}{\rightarrow}} \underset{\xi}{\xi}$ (2) If $\xi, \xi_{1}, \xi_{2}, \cdots \in L_{F}^{0}$ and $\xi_{n} \rightarrow \xi$ in \|\|$_{F}$, then $\xi_{n} \stackrel{F}{\rightarrow} \xi$.

Proof. Suppose that $\xi_{n} \rightarrow \xi$ in $L_{F}$. Then by Proposition (1.3) (3), we see that for any $a>0$, there is an $n_{0} \geqq 1$ such that $E\left[F\left(a\left|\xi-\xi_{n}\right|\right)\right] \leqq 1, n \geqq n_{0}$. Then we have $P\left(\left|\xi-\xi_{n}\right|>\varepsilon\right) \leqq F(a \varepsilon)^{-1}, n \geqq n_{0}$. Combining them, we have our assertion.

Remark. The converse of Proposition (2.2) (1) or (2) is not true. Let $F(x)$ $=x^{2}$, and let $\left\{\xi_{n}\right\}_{n=1}^{\infty}$ be a sequence of ramdom variables such that $P\left(\xi_{n}=n\right)$ $=n^{-2}$ and $P\left(\xi_{n}=0\right)=1-n^{-2}, n=1,2, \cdots$. Then we have $\xi_{n} \stackrel{F}{\rightarrow} 0$, but $\left\{\xi_{n}\right\}_{n=1}^{\infty}$ does not converge to 0 in $L_{F}=L_{F}^{0}=L^{2}(\Omega, \mathscr{B}, P)$.

(2.3) Definition. Let $F_{\imath}:[0, \infty) \rightarrow[0, \infty), i=1,2$, be Young functions. We say that $F_{1} \ll F_{2}$, if $\lim _{x \rightarrow \infty}\left(F_{2}(x) / F_{1}(a x)\right)=\infty$ for all $a>0$.

(2.4) Remark. Let $F:[0, \infty) \rightarrow[0, \infty)$ be a Young function. Let $\tilde{F}(x)=$ $F\left(x^{2}+1\right)-F(1)-2 F^{\prime}(1) x, x \in[0, \infty)$. Then $\tilde{F}:[0, \infty) \rightarrow[0, \infty)$ is also a Young function and $F \ll \tilde{F}$. So for any Young function $F:[0, \infty) \rightarrow[0, \infty)$, there always exists a Young function $\tilde{F}:[0, \infty) \rightarrow[0, \infty)$ with $F \ll \tilde{F}$.

(2.5) Proposition. Let $F_{\imath}:[0, \infty) \rightarrow[0, \infty), i=1,2$, be Young functions with $F_{1} \ll F_{2}$. Then we have the following.

(1) $L_{F_{2}} \subset L_{F_{1}}^{n}$.

(2) If $\xi, \xi_{n} \in L_{F_{2}}, n=1,2, \cdots$, and $\xi_{n} \underset{w}{\stackrel{F_{2}}{\longrightarrow}} \xi, n \rightarrow \infty$, then $\xi_{n} \rightarrow \xi$ in \|\|$_{F_{1}}$.

Proof. Note that

$$
E\left[F_{1}(a|\xi|)\right] \leqq E\left[F_{1}(a(x \wedge|\xi|)]+\left(\sup _{y>x} \frac{F_{1}(a y)}{F_{2}(b y)}\right) E\left[F_{2}(b|\xi|)\right]\right.
$$

for any $a, b, x>0$ and $\xi \in L_{F_{2}}$. So we have our assertion.

Let $a_{f}$ denote the set of all Young functions. Then we have the following. 
(2.6) Proposition. (1) $L^{\infty}(\Omega, \mathscr{B}, P)=\bigcap_{F \in{ }^{\prime \prime}} L_{F}$.

(2) For any Young function $F:[0, \infty) \rightarrow[0, \infty)$, the set $\left(\xi \in L_{F} ;\|\vec{\xi}\|_{F} \leqq 1\right\}$ is a uniformly integrable subset of $L^{1}(\Omega, \mathscr{B}, P)$.

(3) For any uniformly integrable subset $A$ of $L^{1}(\Omega, \mathscr{B}, P)$, there is a Ioung function $F:[0, \infty) \rightarrow[0, \infty)$ such that $A \subset\left\{\xi \in L_{F^{\prime}} ; \| \xi_{\|_{F}} \leqq 1\right\}$

(4) $L^{1}(\Omega, \mathscr{B}, P)=\bigcup_{F \in q} L_{F}$.

Remark. The assertions (2) and (3) are known as "de la Valleé-Poussin's theorem."

Proof. (1) If $\xi \notin L^{\infty}(\Omega, \mathscr{B}, P)$, we see that there is a $C^{1}$-function $G:[0, \infty)$ $\rightarrow[0, \infty)$ such that $E[G(|\xi|)]=\infty$ with $G^{\prime}(0)=0$. Let $f(x)=x+\max _{y \in[0, x]}\left|G^{\prime}(y)\right|$, $x \geqq 0$, and $F(x)=\int_{0}^{x} f(y) d y, x \geqq 0$. Then $F$ is a Young function and $E[F(|\xi|)]$ $=\infty$, and so $\xi \notin L_{F}^{\mathrm{n}}$. Then by Remark (2.4) and Proposition (2.5), we have the assertion (1).

The assertion (2) is obvious from the fact that $\left\{\xi \in L_{F} ;\|\xi\|_{F} \leqq 1\right\}=\left\{\xi \in L^{1}\right.$; $E[F(|\xi|)] \leqq 1\}$ and $\lim _{x \rightarrow \infty}(F(x) / x)=\infty$.

(3) Let $c=\sup _{\xi \in A} E[|\xi|]$. Since $\sup _{\xi \in A} E[|\xi|,|\xi| \geqq n] \rightarrow 0, \quad n \rightarrow \infty$, there are $1 \leqq$ $n_{1}<n_{2}<\cdots$ such that $\sup _{\xi \in 1} E\left[|\xi|,|\xi| \geqq n_{k}\right] \leqq 2^{-k}, k=1,2, \cdots$. Let $n_{0}=0$, and $f:[0, \infty) \rightarrow[0, \infty)$ be given by $f(x)=k-1+\left(n_{k}-n_{k-1}\right)^{-1}\left(x-n_{k-1}\right), x \in\left[n_{k-1}, n_{k}\right)$, $k=1,2, \cdots$. Also let $F(x)=(c+1)^{-1} \int_{0}^{x} f(y) d y, x \geqq 0$. Then $F$ is a Young function and

$$
\begin{aligned}
(c+1) E[F(|\xi|)] & \leqq \sum_{k=1}^{\infty} E\left[|\xi|, k|\xi| \in\left[n_{k-1}, n_{k}\right)\right] \\
& =\sum_{k=1}^{\infty} E\left[|\xi|,|\xi| \geqq n_{k-1}\right] \leqq c-1-1,
\end{aligned}
$$

for any $\xi \in A$. So $A \subset\left\{\xi \in L_{F} ;\|\xi\|_{F} \leqq 1\right\}$ and the assertion (3) is proved.

The assertion (4) is a consequence of the assertions (2) and (3).

(2.7) Lemma. Let $\tau=\tau\left(L^{\infty}(\Omega, \mathscr{B}, P), L^{1}(\Omega, \mathscr{B}, P)\right)$ be the Mackey topology of $L^{\infty}=L^{\infty}(\Omega, \mathscr{B}, P)$. Let $F:[0, \infty) \rightarrow[0, \infty)$ be a Young function and let $\iota: L^{\infty} \rightarrow L_{F}$ be the inclusion map. Then $L^{\infty}, \tau \stackrel{\iota}{\rightarrow} L_{F},\|\|_{F}$ is continuous.

Proof. Let $G:[0, \infty) \rightarrow[0, \infty)$ be the complementary Young function of $F$. Then by Proposition (1.3) (7), we see that

$$
\|\iota(\xi)\|_{F} \leqq \sup \left\{L_{L^{\infty}}\langle\xi, \eta\rangle_{L^{1}} ; \eta \in L_{G}^{\prime \prime},\|\eta\|_{G} \leqq 1\right\} .
$$

By Proposition (2.6) (2), $\left\{\eta \in L_{G} ;\|\eta\|_{G} \leqq 1\right\}$ is a relatively compact convex set 
in $L^{1}$. So we have our assertion.

Remark. It is known that the Mackey topology $\tau$ is the weakest topology in $L^{\infty}(\Omega, \mathscr{B}, P)$ such that the inclusion from $L^{\propto}(\Omega, \mathcal{B}, P)$ into $\left(L_{F},\|\|_{F}\right)$ is continuous for all Young function $F$.

(2.8) Corollary. (1) Let $A$ be a subset of $L^{\infty}(\Omega, \mathscr{B}, P)$. Then $\left(\bigcap_{F \in U} \mathcal{C} l_{F^{\prime}}(A)\right) \cap$ $L^{\infty}(\Omega, \mathscr{B}, P)$ is a closed set in $L^{\infty}, \tau\left(L^{\infty}, L^{1}\right)$.

(2) Let $A$ be a convex subset of $L^{\infty}(\Omega, \mathscr{B}, P)$. Then $\left(\bigcap_{F \in q_{i}} \mathcal{C} l_{F}(A)\right) \cap L^{\infty}(\Omega, \mathscr{B}, P)$ is the closure of $t$ in $L^{\infty}, \tau\left(L^{\infty}, L^{1}\right)$.

Proof. The assertion (1) is obvious from Proposition (2.5) and Lemma (2.7). Therefore in order to prove the assertion (2) it is sufficient to prove that $\left(\bigcap_{F \in q_{l}} \mathcal{C} l_{F}(A)\right) \cap L^{\infty}(\Omega, \mathscr{B}, P)$ is contained in the closure of $A$ in $L^{\infty}, \tau\left(L^{\infty}, L^{1}\right)$. However, since $A$ is convex, Propositions (1.3) (3), (2.5), (2.6) and Hahn-Banach theorem imply it.

Now we have the following Yan's type theorem.

(2.9) Theorem. Let $K$ be a convex set in $L^{\infty}(\Omega, \mathcal{B}, P)$ with $0 \in K$. Then the following three conditions are equivalent.

(1) If $\xi \in L_{+}^{\infty}$ and $P(\xi>0)>0$, then $\{c \cdot \xi ; c>0\} \not \subset \underset{F \in \vartheta}{\bigcap} \mathcal{C} l_{F}\left(K-L_{+}^{\infty}\right)$.

(2) If $A \in \mathscr{B}$ and $P(A)>0$, then $\left\{c \cdot 1_{A} ; c>0\right\} \not \subset \bigcap_{F^{\prime \prime}} \mathcal{C} l_{F}\left(K-L_{+}^{\infty}\right)$.

(3) There is a $\rho \in L^{1}(\Omega, \mathscr{B}, P)$ such that $\rho>0 \quad P$-a.s., $E[\rho]=1$, and $\sup _{\xi \in K} E[\xi \rho]<\infty$.

Proof. (1) $\rightarrow(2)$ is obvious. The proof of $(2) \rightarrow(3)$ is the same as that of Theorem 1 in Ansel-Stricker [1], if we note Corollary (2.8) (2) and the fact that the dual space of $L^{\infty}, \tau\left(L^{\infty}, L^{1}\right)$ is $L^{1}$ (see the proof of Theorem 5.1 in Delbaen [4] also). (3) $\rightarrow(1)$ follows from Theorem (1.5) and Propositions (2.5) and (2.6) (4).

\section{$\S 3$. Existence of Equivalent Martingale Measure}

Let $(\Omega, \mathscr{B}, P)$ be a probability measure, $T>0$, and $\left\{\mathscr{B}_{t}\right\}_{t \in[0, T]}$ be a right continuous increasing sub- $\sigma$-algebras of $\mathscr{B}$. Let $\{X(t) ; t \in[0, T]\}$ be a bounded $\boldsymbol{R}^{d}$-valued $\mathscr{B}_{t}$-adapted process with right continuous paths with probability one. We say that $\xi:[0, T] \times \Omega \rightarrow \boldsymbol{R}^{d}$ is elementary, if there are $n \in \boldsymbol{N}, 0=t_{0} \leqq t_{1}<t_{2}<$ $\cdots<t_{n}=T$, and $\eta_{k}: \Omega \rightarrow \mathbb{R}^{d}, k=1, \cdots, n$, such that

(1) $\eta_{k}$ is $\mathscr{B}_{t_{k-1}}$-measurable and bounded, $k=1, \cdots, n$, and 
(2) $\xi(t)=\sum_{k=1}^{n} \eta_{k} \cdot 1_{\left(t_{k-1}, t_{k}\right]}(t), \quad t \in[0, T]$.

For each elementary process $\xi:[0, T] \times \Omega \rightarrow \boldsymbol{R}^{d}$, let $(\xi \cdot X)(t), t \in[0, T]$, be given by

$$
(\xi \cdot X)(t)=\sum_{k=1}^{n} \eta_{k} \cdot\left(X\left(t \wedge t_{k}\right)-X\left(t \wedge t_{k-1}\right)\right), \quad t \in[0, T] .
$$

Then we see that $(\xi \cdot X)(T) \in L^{\infty}(\Omega, \mathscr{B}, P)$.

Then similarly to the proof of Theorem 2 in Stricker [9], we have the following by using Theorem (2.9).

(3.1) Theorem. Let $K_{0}=\{(\xi \cdot X)(T)$; $\xi$ is an elementary process $\}$. Then the following two conditions are equivalent.

(1) There is a probability measure $Q$ in $(\Omega, \mathscr{B})$ such that the probability measures $P$ and $Q$ are mutually absolutely continuous and $\{X(t) ; t \in[0, T]\}$ is a martingale under the probability measure $Q$.

(2) $L_{+}^{\infty} \cap\left(\bigcap_{F \in \vartheta} C l_{F}\left(K_{0}-L_{+}^{\infty}\right)\right)=\{0\}$.

We say that $\xi:[0, T] \times \Omega \rightarrow \boldsymbol{R}^{d}$ is very simple, if there are $n \in \boldsymbol{N}$, stopping times $\tau_{1}, \tau_{2}, \cdots, \tau_{n}$, and $\eta_{k}: \Omega \rightarrow \boldsymbol{R}^{d}, k=1, \cdots, n$, such that

(1) $\tau_{0}=0 \leqq \tau_{1} \leqq \tau_{2} \leqq \cdots \leqq \tau_{n}=T$ P-a.s.

(2) $\eta_{k}$ is $\mathscr{B}_{\tau_{k-1}}$-measurable and bounded, $k=1, \cdots, n$,

and

(3) $\xi(t)=\sum_{k=1}^{n} \eta_{k} \cdot 1_{\left(\tau_{k-1}, \tau_{k}\right]}(t), t \in[0, T]$,

For very simple process $\xi:[0, T] \times \Omega \rightarrow \boldsymbol{R}^{d}$, let $(\xi \cdot X)(t)$ be given by

$$
(\xi \cdot X)=\sum_{k=1}^{n} \eta_{k} \cdot\left(X\left(t<\tau_{k}\right)-X\left(t \wedge \tau_{k-1}\right)\right) .
$$

Then we see that $(\xi \cdot X)(T) \in L^{\infty}(\Omega, \mathscr{B}, P)$.

Our second result is the following.

(3.2) Theorem. Assume that $X(t), t \subseteq[0, T]$, has continuous paths with probability one and let $K=\{(\xi \cdot X)(T)$; $\xi$ is a very simple process $\}$. Then the following two conditions are equivalent.

(1) There is a probability measure $Q$ in $(\Omega, \mathscr{B})$ such that the probability measures $P$ and $Q$ are mutually absolutely continuous and $\{X(t) ; t \in[0, T]\}$ is a local martingale under the probability measure $Q$.

(2) $L_{+}^{\infty} \cap\left(\bigcap_{F \in \in^{\prime l}} C l_{F}(K)\right)=\{0\}$.

Remark. This theorem is just a corollary to Theorem 5.1 in Delbaen [4]. 
Proof. It is sufficient to prove that the assertion (2) implies $\left.L_{+1}^{\infty}\right\urcorner\left(\bigcap_{F \in U} C l_{F}\left(K-L_{+}^{\infty}\right)\right)=\{0\}$. Suppose that $\xi \neq 0$ and $\xi \in L_{+}^{\infty} \cap\left(\bigcap_{F \in a_{J}} C l_{F}\left(K-L_{+}^{\infty}\right)\right)$. Then by the similar argument in the proof of Theorem 3 in Stricker [11] shows that there is an $\eta \in \underset{F \in \eta_{l}}{\cap} \mathcal{C} l_{F}(K)$ with $\xi \wedge 1 \leqq \eta \leqq 1$. This contradicts the assertion (2). So we have done it.

\section{References}

[1] Ansel, J.-P. and Stricker, C., Quelques remarques sur un théorem de Yan, Seminaire de Probabilites XXIV, Lect. Notes Math., 1426, pp. 266-274, Springer, BerlinHeidelberg-New York, 1990.

[2] Dalang, R.C., Morton, A. and Willinger, W., Equivalent Martingale Measures and No-Arbitrage in Stochastic Securities Market Models, Stochastic and Stochastic Reports, 29 (1990), 185-201.

[3] Delbaen, F., Representing martingale measures when asset prices are continuous and bounded, Mathematical Finance, 2 (1992), 107-130.

[4] Delbaen, F. and Schachermayer, W., Arbitrage and free lunch with bounded risk for unbounded continuous processes, Preprint.

[5] Delbaen, F. and Schachermayer, W., A General Version of the Fundamental Theorem of Asset Pricing, Preprint.

[6] Föllmer, H., Probabilistic Aspects of Option, Discussion Paper No. B-202, Information und die Koordination wirshaftlicher Aktivität, Universität Bonn.

[7] Harrison, J.M. and Kreps, D. M., Martingales and Arbitrage in Multiperiod Securities Market. Journal of Economic Theory, 20 (1979), 381-408.

[8] Krasnosel'skii, M. A. and Rutickiǐ, Ya.B., Convex functions and Orlicz spaces, Gordon and Breach 1961, New York.

[9] Schachermayer, W., A Hilbert space proof of the fundamental theorem of asset pricing in finite discrete time, Insurance Mathematics and Economics, 11 (1992), 1-9.

[10] Schachermayer, W., Martingale measures for discrete time with infinite horizon, Preprint.

[11] Stricker, C., $\Lambda$ rbitrage et Lois de Martingale. Ann. Inst. Henri Poincaré, 26 (1990), 451-460.

[12] Yan, J.A., Caractérisation d'une classe d'ensembles convexes de $L^{1}$ ou $H^{1}, S e$ minaire de Probabilites XIV, Lect. Notes Math., 784, pp. 220-222, Springer, BerlinHeidelberg-New York, 1980.

[13] Zaanen, A. C.. Riesz spaces II, North-Holland, Amsterdam, 1983. 